\title{
MODELOS DE PREVISÃO DO REBANHO BOVINO BASEADOS NAS SÉRIES TEMPORAIS DO ESPÍRITO SANTO E DO BRASIL
}

\author{
GIANNOTTI, Juliana Di Giorgio ${ }^{1}$ \\ OLIVEIRA, Allison Queiroz de ${ }^{2}$
}

RESUMO: A metodologia de séries temporais foi utilizada com o objetivo de ajustar modelos de tendência e previsão para o rebanho bovino do Brasil e do estado do Espírito Santo. Coletou-se a série de dados anuais contendo as informações sobre o número de cabeças de bovinos, entre os anos de 1974 a 2017, para o Brasil e Espírito Santo da Pesquisa Pecuária Municipal (PPM) do Instituto Brasileiro de Geografia e Estatística (IBGE). O modelo de tendência quadrática ajustado com fator de amortecimento 0,25 foi o mais indicado para a previsão do efetivo de todo o território brasileiro com coeficiente de determinação igual a 0,9928, enquanto que somente para o Espírito Santo o modelo de tendência quadrática ajustado pelo método de médias móveis em agrupamento de cinco anos foi o mais adequado para realizar a previsão do efetivo nos próximos anos, com coeficiente de determinação igual a 0,7546 .

Palavras-chave: Séries Históricas. Modelos De Previsão. População Bovina.

\section{BEEF CATTLE FORECAST MODELS BASED ON THE ESPÍRITO SANTO AND BRASIL TIME SERIES}

SUMMARY: The time series methodology was used with the main of adjusted trend and forecasting models to Brazilian and Espírito Santo State beef cattle. It was gathered the annual series data with information about the number of beef cattle between 1974 and 2017 years, to Brazil and Espírito Santo from IBGE's Municipal Livestock Research. The quadratic trend model adjusted with a 0,25 damping factor could be the best one to forecast of beef cattle to all Brazilian territory with the value of coefficient of determination was 0,9928, while the trend quadratic model adjusted by the moving averages method and clustered from five years was the most adequate to realize the prediction of livestock population to next years, with the value of coefficient of determination was 0,7546 .

Keywords: Historical Series. Forecasting Models. Livestock Populations.

\section{INTRODUÇÃO}

A bovinocultura tem fundamental importância econômica para o Brasil uma vez que o país tem consolidada sua posição de maior exportador mundial de carne bovina (FAO, 2018) e detém o segundo maior rebanho bovino mundial atrás da Índia e seguido pela China e Estados Unidos, respectivamente (FAO, 2017).

A criação de bovinos está distribuída por todo o território brasileiro, sendo que o estado de Mato Grosso concentra $14 \%$ da produção e é o maior produtor brasileiro, seguido de Goiás com 11\%, Minas Gerais, Mato Grosso do Sul e Pará todos concentrando 10\% da bovinocultura

\footnotetext{
${ }^{1}$ Professor Associado - Departamento de Engenharia Rural - Área Estatística -

Universidade Federal do Espírito Santo

${ }^{2}$ Graduando - Zootecnia - Universidade Federal do Espírito Santo
} 
nacional. O estado do Espírito Santo ocupa a $17^{\underline{a}}$ posição no ranking brasileiro, sendo responsável por aproximadamente $1 \%$ da produção nacional (IBGE-PPM, 2018).

Embora no âmbito nacional o Espírito Santo não tenha participação expressiva na criação de bovinos, em nível estadual a pecuária leiteira e de corte do estado abrangem 32 mil produtores, sendo 18 mil de leite e 14 mil de corte os quais são na sua maioria destinados ao mercado interno e tem participação de $13 \%$ no valor bruto da produção agropecuária do estado, evidenciando deste modo a importância do setor para o estado (INCAPER, 2018).

Dados da Pesquisa Pecuária Municipal do Instituto Brasileiro de Geografia e Estatística, PPM-IBGE, apontam para um aumento do rebanho bovino do estado ao longo dos anos (IBGEPPM, 2018). Porém uma análise mais detalhada dos dados do efetivo bovino que contemple aspectos como a tendência de crescimento, possíveis quedas em anos específicos e uma previsão futura do número de cabeças para o estado pode contribuir para uma melhor gestão dos investimentos e nortear políticas de desenvolvimento para o setor.

A fim de obter estes resultados pode-se aplicar a metodologia de séries temporais, que consiste em obter um conjunto de dados numéricos coletados ao longo do tempo e, a partir de dados do passado realizar uma previsão futura da variável em questão (LEVINE, 2012). A característica mais importante deste tipo de dados é a dependência das observações vizinhas e o interesse recai sobre a análise e modelagem desta dependência (EHLERS, 2007). O estudo de séries temporais como método de modelagem e predição pode auxiliar na identificação das marcas que a bovinocultura no estado do Espírito Santo e no Brasil como um todo tende a alcançar em anos futuros.

Assim, o objetivo deste trabalho foi a análise temporal das séries referentes ao efetivo do rebanho bovino do Espírito Santo e do Brasil, a fim de, baseado neses dados históricos, analisar a tendência e realizar predições com relação ao tamanho do rebanho efetivo nos próximos anos.

\section{MATERIAL E MÉTODO}

As séries temporais utilizadas foram obtidas do banco de dados SIDRA (Sistema IBGE de Recuperação Automática) da PPM-IBGE (IBGE-PPM, 2018), e são referentes à variável número de cabeças de bovinos para o Brasil e para o estado do Espírito Santo no período compreendido entre 1974 e 2017.

Tanto para os dados do Brasil quanto aos do Espírito Santo, realizou-se em um primeiro momento o ajuste da série de dados por dois métodos de acordo com a metodologia proposta por Levine et al. (2012): método de médias móveis (MM), que consiste em uma série de médias aritméticas calculadas em determinada sequência ao longo do tempo, neste método os anos foram 
agrupados em uma sequência de cinco anos; e o método de ajuste exponencial (AE), onde as médias móveis são exponencialmente ponderadas, tendo como base o fator de amortecimento $\mathrm{W}$ $=0,25$.

Em seguida, após a série ter sido ajustada, calculou-se a regressão múltipla a fim de realizar predições por meio do modelo de tendência quadrática, dado por:

$$
Y_{i}=\beta_{0}+\beta_{1} X_{i}+\beta_{2} X_{i}^{2}+\varepsilon_{i}
$$

Os dados foram tabulados e os anos da série temporal codificados em uma sequência pertinente de zero a 43, sendo o zero correspondente ao ano de 1974 e o 43 ao ano de2017. Todas as análises estatísticas dos dados foram realizadas no programa computacional de acesso livre RStudio (RStudio Development Core Team, 2018).

\section{RESULTADO E DISCUSSÃO}

Na Figura 1, o gráfico de boxplot indica a distribuição do efetivo de bovinos no território brasileiro ao longo dos anos. Observa-se que $25 \%$ do número de cabeças ao longo dos anos encontra-se até o efetivo de 128.230.649 de cabeças, enquanto 75\% da mesma variável é menor ou igual a 204.711.541. A média do efetivo no período compreendido da série é de 162.630.153. O menor valor encontrado foi de 92.495 .364 cabeças referente ao ano de 1974 e o maior valor foi 218.199.581 de cabeças para o ano de 2016.

Figura 1: Boxplot do efetivo de bovinos no Brasil

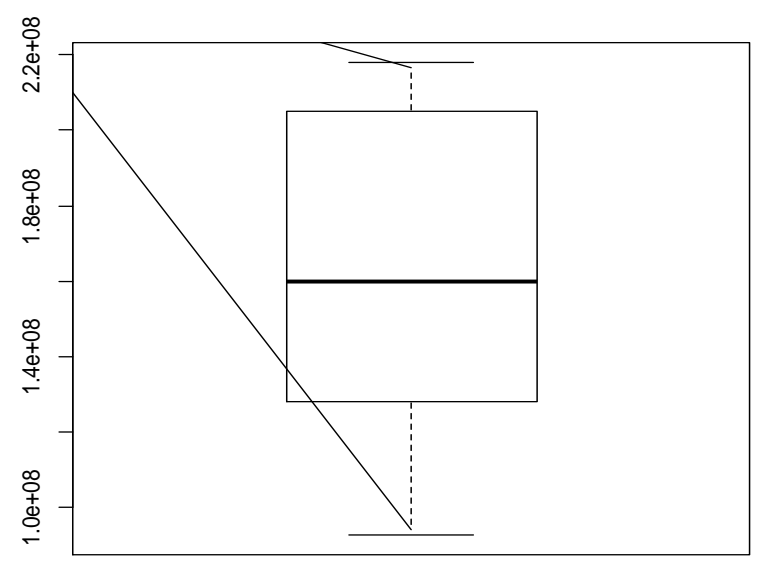

Fonte: Elaborado pelos autores 
Na Figura 2 estão apresentados, para o efetivo de bovinos no Brasil, os gráficos de dispersão e a curva de tendência para os modelos quadráticos com ajuste exponencial e fator de amortecimento de 0,25, de médias móveis por agrupamento de cinco anos e o isento de ajuste. Sendo estes ajustes os que apresentaram melhor suavização dos dados neste modelo.

Figura 2: Gráficos do modelo de previsão de tendência quadrática, para o Brasil, correlata com cada ajuste realizado.
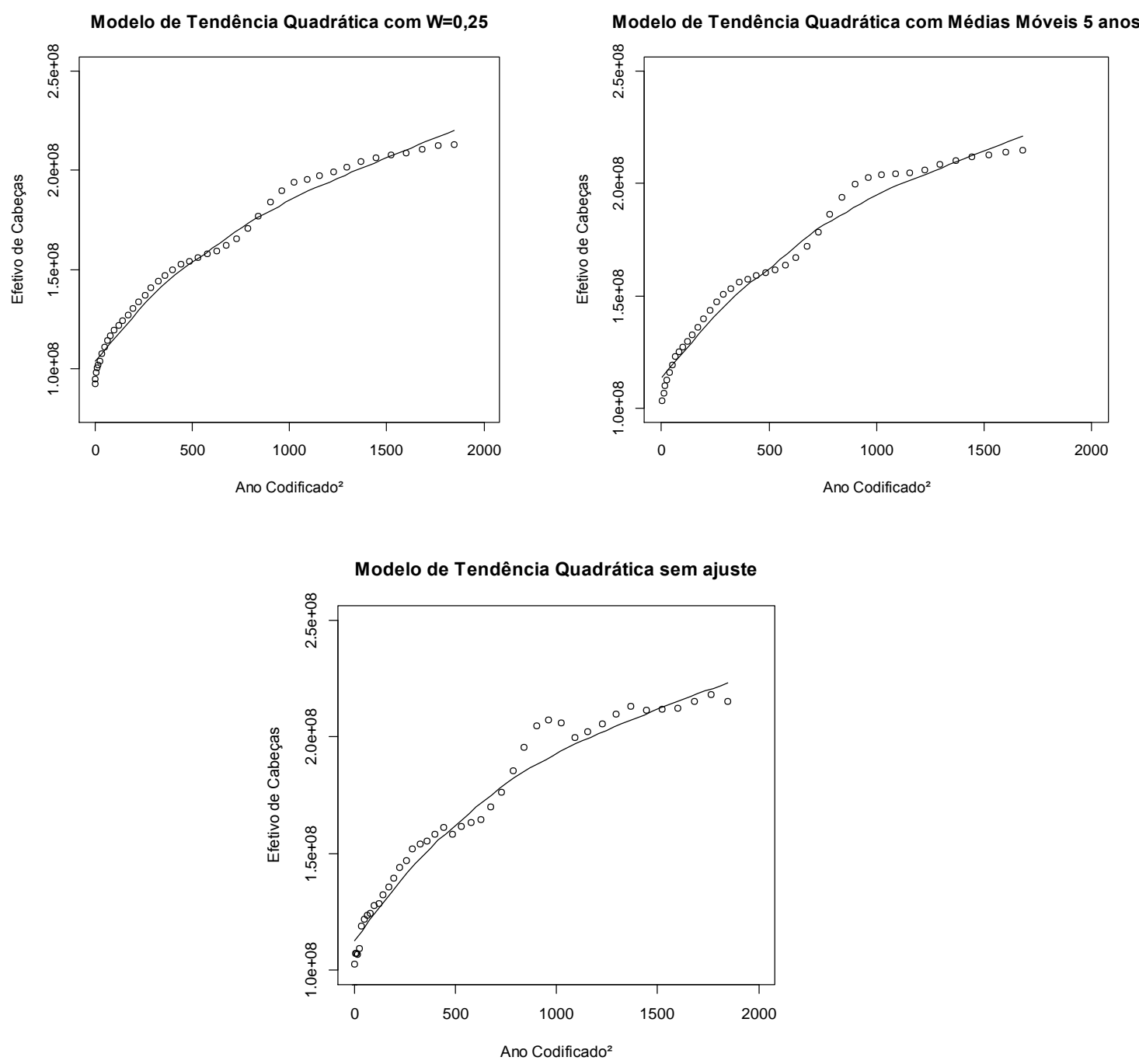

Fonte: Elaborado pelos autores

Na Tabela 1 encontram-se, para a série temporal do Brasil, os modelos quadráticos ajustados com valores codificados de zero a 43 como a variável X (ano codificado) e os dados de cabeça bovina como a variável Y, como também o modelo não ajustado. 
Tabela 1: Modelos de regressão para o efetivo bovino do Brasil, por tipo de ajuste.

\begin{tabular}{|c|c|c|c|c|}
\hline \multirow{2}{*}{ Modelo } & \multicolumn{4}{|c|}{ Estatísticas } \\
\hline & Equação & $\mathbf{R}^{2}$ & $\mathbf{R}^{2}$ ajustado & $\mathbf{F}_{\text {calculado }}$ \\
\hline Quadrático sem ajuste & $Y_{i}=95232993+3408208 x-9430 x^{2}$ & 0,979 & 0,978 & $966,4 * * *$ \\
\hline Quadrático $\mathrm{W}=0,25$ & $Y_{i}=90698228+2870533 x+3209 x^{2}$ & 0,993 & 0,993 & $2959,0 * * *$ \\
\hline Quadrático MM cinco anos & $Y_{i}=96092679+3216613 x-3593 x^{2}$ & 0,986 & 0,985 & $1267,0 * * *$ \\
\hline
\end{tabular}

Fonte: Elaborado pelos autores

*** significativo a 0,001 de probabilidade pelo teste $\mathrm{F}$ da análise de variância

Os resultados presentes na tabela 1 indicam uma previsão 244.524.171 de cabeças para o ano de 2025, sendo este codificado pelo número 51, quando se utiliza o modelo quadrático sem ajuste. Para os modelos quadráticos com ajuste por fator de amortecimento 0,25 e com ajuste de médias móveis por agrupamento de cinco anos a previsão para o ano de 2025 foi respectivamente de 245.442.020 cabeças e 250.794.549 de cabeças em toda extensão territorial brasileira.

Os gráficos de dispersão (Figura 2) estão em consonância com o crescimento sugerido pelas equações (Tabela 1), em cada um dos ajustes, para o tamanho do rebanho no Brasil. Os coeficientes de determinação $\left(\mathrm{R}^{2}\right)$ dos modelos propostos são considerados altos, entretanto o modelo com ajuste por fator de amortecimento 0,25 teve o maior coeficiente e apresentou melhor suavidade da curva em meio aos dados utilizados, podendo ser indicado como o melhor para a previsão do efetivo brasileiro.

Strassburg et al. (2014) em um estudo sobre como as demandas futuras de produção vegetal e animal irão impactar no meio ambiente, utilizaram quatro modelos, os quais incluíram dados climáticos, a fim de obter estimativas sobre o total de rebanho brasileiro necessário para atender a demanda projetada. Assim, considerando esses modelos as estimativas do efetivo de rebanho para o Brasil encontradas por estes autores ficaram entre 292.769.702 e 377.444.819 para o ano de 2040.

Considerando o modelo quadrático com ponderação 0,25 encontrado neste trabalho, a previsão do efetivo de rebanho para o Brasil no ano de 2040 será 294.131.810 valor que pertence ao intervalo encontrado por Strassgurg et al. (2014), estando assim em conformidade com o encontrado por esses autores.

Silva et al. (2018) trabalharam com modelos considerando diferentes cenários ambientais os quais foram desde as projeções do aumento das pastagens pelo desmatamento avançando sobre a vegetação natural, até os modelos cujas metas são a intensificação das pastagens com o uso de animais confinados alimentados com concentrados e suplementos proteicos e priorizando a diminuição da degradação ambiental. Nestes cenários a média obtida por Silva et al. (2018) do 
efetivo bovino brasileiro para o período de 2020 a 2030 variou de 204.520 .000 a 205.700 .00 de cabeças.

Arantes et al. (2018) em um estudo que uniu dados do censo agrícola e de sensoriamento remoto sobre o potencial de intensificação da pecuária no Brasil, verificaram que há um potencial de aumento da lotação bovina para 2,63 unidades animais por hectare, em média, considerando todo o território brasileiro. Tal resultado converge com os do presente trabalho, uma vez que com a previsão do aumento do efetivo bovino pode haver a necessidade da intensificação da lotação territorial pelo rebanho bovino.

Para o modelo quadrático com ponderação 0,25 , obtido neste trabalho, a previsão do efetivo bovino brasileiro no ano de 2020 será de 229.532.990, esse valor foi maior do que o obtido pelos autores Silva et al. (2018). Tal resultado pode ser explicado em virtude dos modelos utilizados por esses autores considerarem diversas variáveis ambientais que, no modelo por eles apresentado possam atuar como um limitador do crescimento do número de cabeças de bovinos para o cenário brasileiro.

Na Figura 3, é apresentado o gráfico de boxplot do efetivo de bovinos no estado do Espírito Santo ao longo dos anos. De acordo com a análise do boxplot verifica-se que $25 \%$ do número de cabeças ao longo do tempo encontram-se até o efetivo de 1.791 .435 de cabeças e $75 \%$ do efetivo é menor ou igual a 2.130.162. A média da variável em estudo no período analisado é de 1.952.177. O mínimo encontrado entre os valores foi de 1.664 .627 de cabeças para o ano de 1987 e o maior valor foi de 2.313 .445 para o ano de 2013.

Figura 3: Boxplot do efetivo de bovinos no Espírito Santo

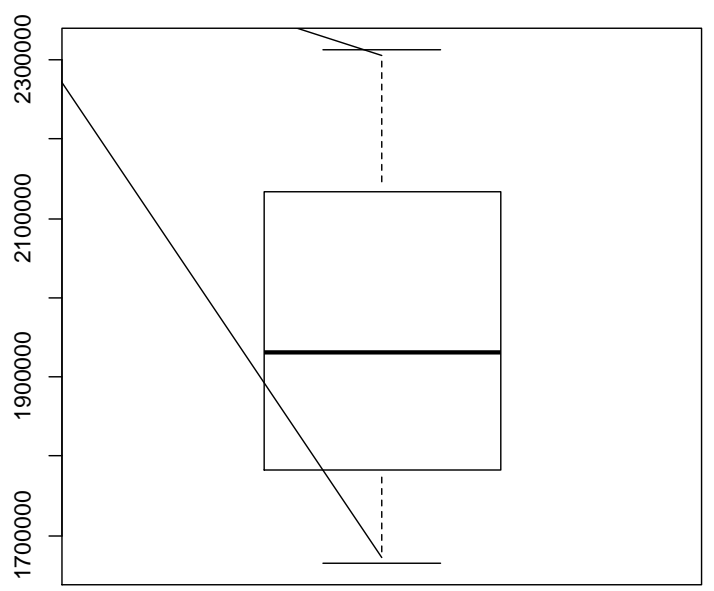

Fonte: Elaborado pelos autores 
Na Figura 4 estão apresentados, para o efetivo de bovinos no estado do Espírito Santo, os gráficos de dispersão e a curva para o modelo de tendência quadrática em função do ajuste exponencial com o fator de amortecimento de 0,25 , de médias móveis por agrupamento de cinco anos e o isento de ajuste.

Figura 4: Gráficos do modelo de previsão de tendência quadrática, para o estado do Espírito Santo, correlata com cada ajuste realizado.
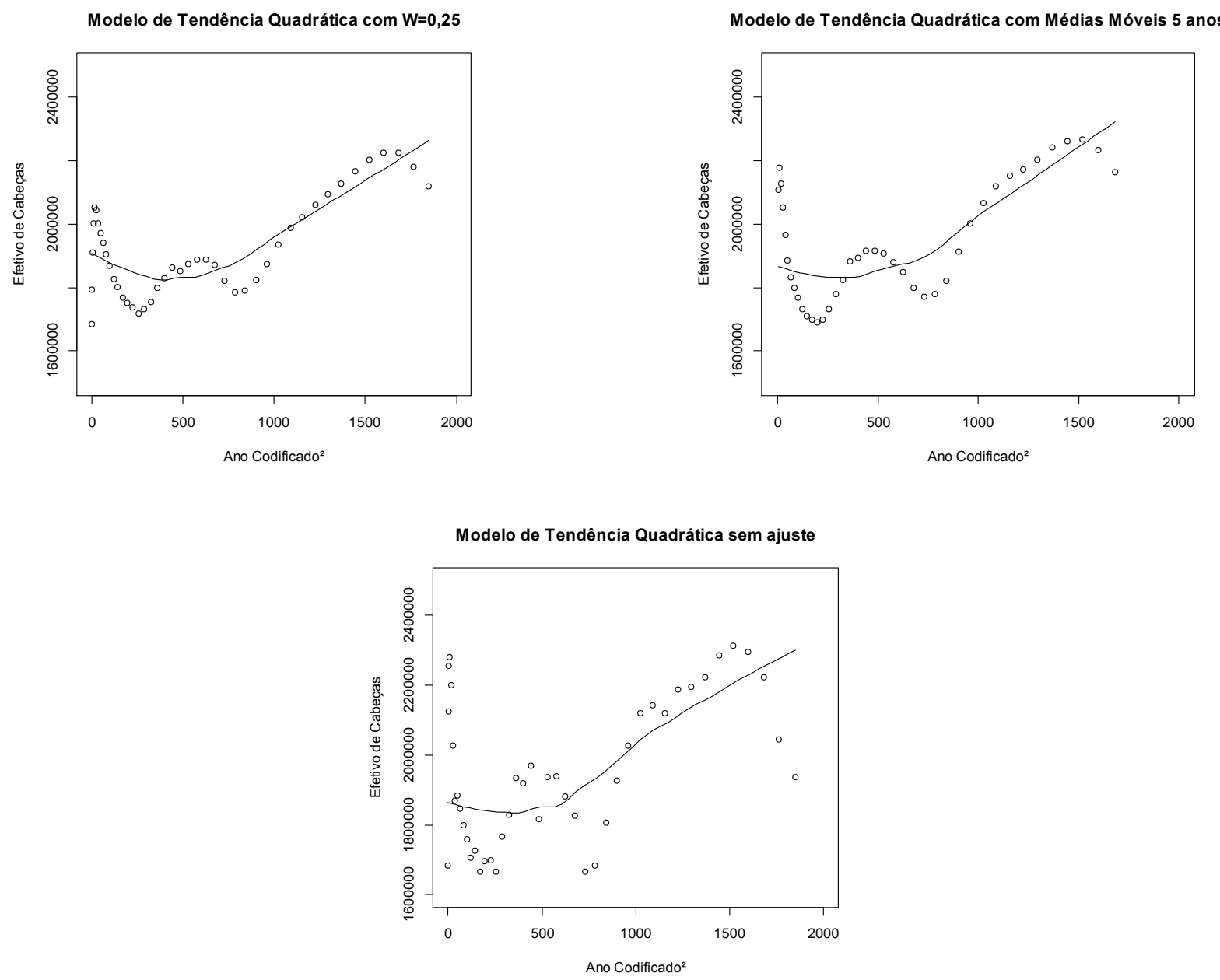

Fonte: Elaborado pelos autores

Na Tabela 2 são apresentados, para a série do Espírito Santo, os modelos quadráticos ajustados com valores codificados de zero a 43 como a variável X (ano codificado) e os dados de cabeça bovina como a variável Y, como também o modelo não ajustado. 
Tabela 2: Modelos de regressão para o efetivo bovino do Espírito Santo, por tipo de ajuste.

\begin{tabular}{l|l|c|r|c}
\hline \multirow{2}{*}{\multicolumn{1}{c|}{ Modelo }} & \multicolumn{4}{c}{ Estatísticas } \\
\cline { 2 - 5 } & \multicolumn{1}{|c|}{ Equação } & $\mathbf{R}^{\mathbf{2}}$ & $\mathbf{R}^{\mathbf{2}}$ ajustado & $\mathbf{F}_{\text {calculado }}$ \\
\hline Quadrático sem ajuste & $\mathrm{Y}_{\mathrm{i}}=2057923,9-27728,8 \mathrm{x}+786,6 \mathrm{x}^{\mathbf{2}}$ & 0,4465 & 0,4195 & $16,54 * * *$ \\
\hline Quadrático W=0,25 & $\mathrm{Y}_{\mathrm{i}=1973039,1-20462,4 \mathrm{x}+624,4 \mathrm{x}^{\mathbf{2}}}$ & 0,6635 & 0,6471 & $40,42^{* * *}$ \\
\hline Quadrático MM cinco anos & $\mathrm{Y}_{\mathrm{i}}=2152577,4-40753,2 \mathrm{x}+1122,3 \mathrm{x}^{\mathbf{2}}$ & 0,7671 & 0,7546 & $60,95 * * *$ \\
\hline
\end{tabular}

Fonte: Elaborado pelos autores

*** significativo a 0,001 de probabilidade pelo teste $\mathrm{F}$ da análise de variância

Os resultados presentes na Tabela 2, indicam previsão de 2.689.702 de cabeças para o ano de 2025 quando se utiliza o modelo quadrático sem ajuste. Para os modelos quadráticos com ajuste por fator de amortecimento 0,25 e com ajuste de médias móveis por agrupamento de cinco anos a previsão para o ano de 2025 foi respectivamente de 2.553 .521 e 2.993.267 de cabeças para o estado.

Observando os gráficos da Figura 4, percebe-se que os mesmos indicam queda do tamanho do efetivo de bovinos no estado a partir do ano de 2015, provocada, possivelmente, pelas condições climáticas adversas, principalmente a estiagem, ocorridas no estado do Espírito Santo nos anos de 2014 e 2015. As equações referentes a cada modelo, presentes na Tabela 2, apresentam uma tendência de crescimento do efetivo bovino capixaba, assim, essa queda observada nos dados a partir de 2015 foi suavizada pelo histórico anterior de crescimento dos dados. Os coeficientes de determinação $\left(\mathrm{R}^{2}\right)$ dos modelos propostos são considerados moderados, entretanto o ajuste por médias móveis com agrupamento de cinco anos teve o maior coeficiente em relação aos outros dois, e indica que um percentual superior a $76 \%$ da variação ocorrida no efetivo do rebanho do Espírito Santo pode ser explicada pela tendência quadrática ao longo da série temporal. Sendo assim, pode-se recomendar o modelo de médias móveis para se realizar a previsão futura do efetivo bovino no estado.

Galeano et al. (2016) no estudo da síntese da produção agropecuária do Espírito Santo utilizaram séries históricas do IBGE e do AnualPec (anuário de pecuária brasileira), entre os anos de 2000 a 2014, e observaram o aumento no efetivo bovino do estado no período. As informações apresentadas pelos autores estão em coerência com o que foi encontrado neste trabalho, uma vez que mesmo tendo uma queda em anos específicos, observa-se uma tendência de aumento do efetivo bovino no estado do Espírito Santo.

De maneira geral a tendência de crescimento do efetivo bovino no Brasil e no Espírito Santo, observada pelos modelos obtidos neste trabalho, apresentaram resultados significativos e indicaram que a variação ocorrida no número de cabeças pode ser explicada pela relação quadrática entre as variáveis. Pontua-se ainda que tal tendência está de acordo com a literatura, 
conforme exposto anteriormente e, com a modelagem recente encontrada pela FAO e Banco Mundial, a qual prevê aumentos grandes e rápidos nas populações de bovinos, principalmente em países em desenvolvimento da América Latina, Caribe, Leste e Sul da Ásia (TURK, 2016).

\section{CONCLUSÃO}

O modelo de tendência quadrática ajustado com fator de amortecimento 0,25 se apresentou como o mais indicado para realizar a predição do efetivo bovino brasileiro, pois apresenta tanto valores futuros em conformidade com outros modelos presentes na literatura, como o maior coeficiente de determinação frente às análises realizadas.

Para o estado do Espírito Santo, o modelo de tendência quadrática ajustado pelo método de médias móveis com agrupamento de cinco anos pode ser considerado o mais apropriado uma vez que apresenta o maior coeficiente de determinação dentre os modelos ajustados.

\section{REFERÊNCIAS}

ARANTES, A.E.et al . Livestock intensification potential in Brazil based on agricultural census and satellite data analysis. Pesq. agropec. bras., Brasília , v. 53, n. 9, p. 1053-1060, Sept. 2018 . Disponível em: <http://www.scielo.br/scielo.php?script=sci_arttext\&pid=S0100204X2018000901053\&lng=en\&nrm=iso>. Accesso em: 18 outubro 2019.

\section{EHLERS, R. S. Análise de Séries Temporais.}

http://conteudo.icmc.usp.br/pessoas/ehlers/stemp/stemp.pdf (Acesso em 07 de agosto de 2018). FAO. Food And Agriculture Organization Of The United Nations (FAO). FAO, 2017.

Disponível em: <http://www.fao.org/faostat/en/\#data/QA >. Acesso em: 14 nov. 2018.

FAO. Food And Agriculture Organization Of The United Nations (FAO). FAO, 2018.

Disponível em: <http://www.fao.org/brasil/noticias/detail-events/en/c/1145212/>. Acesso em: 14 nov. 2018.

GALEANO, E. A. V.et al. Síntese da produção agropecuária do Espírito Santo 2013/2014. Vitória, ES: Incaper, 2016. 116p.

IBGE-PPM. Instituto Brasileiro de Geografia e Estatística. Pesquisa Pecuária Municipal, 2018. Disponível em: 〈http://www.sidra.ibge.gov.br〉. Acesso em: 6 novembro 2018.

INCAPER. Instituto Capixaba de Pesquisa, Assistência Técnica e Extensão Rural. Pecuária. Disponível em: < https://incaper.es.gov.br/pecuaria > Acesso em: 14 novembro 2018.

LEVINE, D.M. et al. Estatística: teoria e aplicações. 6 ed. Rio de Janeiro: LCT, 2012. 804p.

MORETIN, P.A.; TOLOI, C.M.C. Análise de séries temporais. São Paulo: Edgard Blücher, 2004. 535p. 
R DEVELOPMENT CORE TEAM. R: A language and environment for statistical computing, 2013. R Foundation for Statistical Computing, Vienna, Austria. ISBN 3-900051-070, URL <http://www.R-project.org/>. Acesso em: 03 de maio 2013.

SILVA, R. de O.et al. The role of agricultural intensification in Brazil's Nationally Determined Contribution on emissions mitigation. Agricultural Systems. v. 161, 2018, p. 102-112. https://www.sciencedirect.com/science/article/pii/S0308521X17307655 Acesso em: 28 de fevereiro de 2019.

STRASSBURG, B.B.N.et al. When enough should be enough: Improving the use of current agricultural lands could meet production demands and spare natural habitats in Brazil, Global Environmental Change. v. 28, 2014, Pages 84-97, https://doi.org/10.1016/j.gloenvcha.2014.06.001. (http://www.sciencedirect.com/science/article/pii/S0959378014001046) Acesso em: 25 de fevereiro de 2019.

TURK, J. Meeting projected food demands by 2050: Understanding and enhancing the role of grazing ruminants, Journal of Animal Science. v. 94, Issue suppl_6, 1 October 2016, Pages 5362, https://doi.org/10.2527/jas.2016-0547 